\title{
A wall-wake model for the turbulence structure of boundary layers. Part 2. Further experimental support
}

\author{
By IVAN MARUŠIĆ AND A. E. PERRY \\ Department of Mechanical and Manufacturing Engineering, University of Melbourne, Parkville, \\ Victoria 3052, Australia
}

(Received 14 January 1994 and in revised form 22 April 1995)

In Part 1 an extension of the attached eddy hypothesis was developed and applied to equilibrium pressure gradient turbulent boundary layers. In this paper the formulation is applied to data measured by the authors from non-equilibrium layers and agreement with the extended theory is encouraging. Also power spectra of the Reynolds stresses as developed from the extended theory compare favourably with experiment. The experimental data include a check of cone-angle effects by using a flying hot wire.

\section{Introduction}

In Part 1 (Perry \& Marušic 1995), an extension of the attached eddy hypothesis of Townsend (1976) has been applied to turbulent boundary layers developing in pressure gradients. Data used were from adverse and favourable pressure gradient equilibrium layers where the Coles wake factor, $\Pi$, is approximately invariant with streamwise distance. It has been shown by Perry, Marušic \& Li (1994) that it is possible that with such layers there is a one-to-one correspondence with the non-dimensional velocity defect distribution and the non-dimensional shear stress distribution. Both are approximately fixed by the parameter $\Pi$. However, with non-equilibrium layers measured by the authors, this condition breaks down because the streamwise derivative of the Coles wake factor plays a significant role in the momentum balance. The relevant parameter $|\sigma|$ defined in Part 1 for the authors' data is of order unity. Using the new wall-wake attached eddy model developed in Part 1 with the same eddy shapes, encouraging agreement has been found for the Reynolds stress distributions and turbulence power spectral densities. The part played by Kolmogorov motions is included here in the model.

The data presented are from measurements taken at a series of streamwise positions for different flow cases. To the authors' knowledge, these are the only data measured for non-equilibrium adverse pressure gradient layers which give mean flow, Reynolds shear stresses and all three components of the Reynolds normal stresses together with measured spectra. One needs these data for a proper evaluation of the attached eddy hypothesis.

Notation used is identical to that used in Part 1 but important variables are defined again in table 1. 


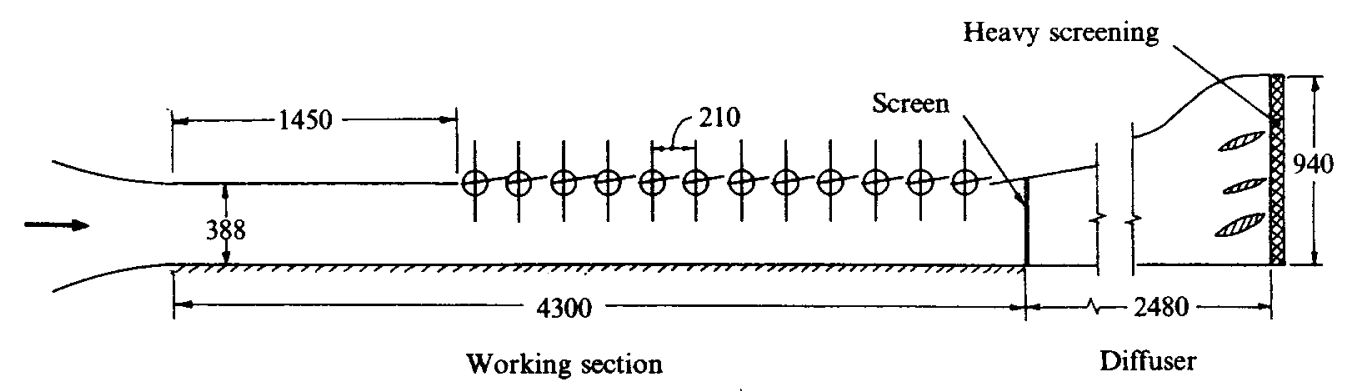

FIGURE 1. Details of working section (dimensions in $\mathrm{mm}$ ).

\section{Experimental apparatus and methods}

In this section, details of the experimental procedure, methods and apparatus are presented. Relevant definitions are also given.

\subsection{Wind tunnel and hot-wire anemometry}

The wind tunnel used in this investigation is of an open-return blower type. It consists of a contraction with area ratio 8.9:1 leading to the inlet of a $4.3 \mathrm{~m}$ long working section with cross-sectional dimensions of $940 \times 388 \mathrm{~mm}$. The free-stream velocity at the inlet of the working section can be varied between $2-35 \mathrm{~m} \mathrm{~s}^{-1}$, while the free-stream turbulence intensity is of the order of about $0.3 \%$ for the operating conditions. A side view of the working section is shown in figure 1 . It consists of a smooth wall whose finished surface is a polished acrylic laminate and is fitted with 68 pressure tappings spaced along its streamwise centreline. The pressure gradient imposed along the smooth wall was established by heavily screening the rear of the diffuser section and by varying the angles of twelve adjustable louvres, which made up part of the roof of the working section. The louvres act as a control for the amount of air being bled from the working section and the location of the bleeding. (The same principal was used by Clauser 1954.) An additional screen, located at the outlet of the working section, was found to greatly aid in controlling the overpressure in the working section. The initial $1.45 \mathrm{~m}$ of the working section consisted of a flat roof which can be tilted at small angles. This arrangement led to flow conditions which were originally in a state of approximately zero pressure gradient which were then perturbed by an adverse pressure gradient.

Central to the design of the working section was the need to accommodate a flying hot-wire facility, which is mounted above the working section and is described in Watmuff, Perry \& Chong (1983). Each of the louvres was installed in two pieces, being cantilevered from both sides such that a $56 \mathrm{~mm}$ gap remained close to the centreline of the tunnel. This slot allowed room for the 'sting' of the flying hot-wire mechanism to pass through and travel along the length of the working section. Tests for two-dimensionality of the flow revealed significant secondary flows being introduced because of this streamwise slot. These tests involved measuring the streamwise and spanwise mean velocities ( $U$ and $V$ respectively), both near the wall and in the free stream, across the spanwise extent of the working section using an X-wire probe. The problem of secondary flows was overcome by using a series of carefully fared sealing gates across the slots which were prised open by a 'cow-catcher' mechanism mounted around the sting. The prising cage is isolated from the hot-wire sting in order to avoid transfer of vibration, and overall the mechanism was found to work quite smoothly and could comfortably operate at a flying speed of $3 \mathrm{~m} \mathrm{~s}^{-1}$. Further 
details of the working section, two-dimensionality tests and the sealing mechanism are given by Marušic (1991).

All turbulence measurements were made with X-wires constructed with $5 \mu \mathrm{m}$ diameter platinum Wollaston wire, etched nominally to $1.0 \mathrm{~mm}$. The wires were separated by a distance of $1.0 \mathrm{~mm}$ and were nominally attached at $\pm 45^{\circ}$ to the streamwise direction. The $\mathrm{X}$-wire probe was held in a chuck which allowed accurate rotation through $90^{\circ}$, allowing measurements to be taken in both the $(x, z)$ - and $(x, y)$-planes where $x$ is the streamwise, $y$ the spanwise and $z$ the wall-normal coordinates. Constant temperature hot-wire anemometers were used for all measurements and were operated at a resistance ratio of 2.0 . The anemometry and the nonlinear dynamic calibration procedure used in the study are similar to those described by Perry (1982).

\subsection{Measuring details and definitions}

Mean flow profiles were measured using a Pitot-static probe in which the total and static tubes were located at the same distance from the wall. Pressure differences were measured with a MKS Baratron 170M-6C manometer with a type $310 \mathrm{BH}-1$ sensor. An additional Pitot-static tube located at the beginning of the working section was used to obtain a reference velocity $U_{\infty}$. This reference condition was used to define a coefficient of pressure $C_{P}$, where

$$
C_{P}=\frac{P-P_{\infty}}{\frac{1}{2} \rho U_{\infty}^{2}}=1-\left(\frac{U_{1}}{U_{\infty}}\right)^{2}
$$

where $\rho$ is fluid density and $P$ is static pressure.

The wall shear velocity $U_{\tau}$ was determined using a Clauser chart and Preston tube method, which were found to agree to within $2 \%$ for all cases. All results to be presented use the Clauser chart estimate of $U_{\tau}$.

Turbulence signals were sampled on-line to a PC using a 12-bit A-D converter (Analog Devices RTI-860). For the broadband turbulence measurements, the hotwire signals were low-pass filtered at $15.8 \mathrm{kHz}$ with a fourth-order Butterworth filter. In order to approach convergence of the Reynolds stresses 32000 samples were taken at a rate of $200 \mathrm{~Hz}$ for the stationary case, while for the flying measurements 60000 samples were taken at a rate of approximately $3000 \mathrm{~Hz}$ (determined by flying speed) over a $55 \mathrm{~mm}$ range about the measuring station. (This corresponded to 1000 sled passes.) The choice of these criteria was made in the light of extensive convergence tests which have been reported by Marušic (1991).

Calibrated wires hold their calibration for only a limited period and one should make maximum use of them for measuring stresses. Power spectral densities were calculated from dynamically matched but uncalibrated X-wire signals by using a FFTalgorithm. Perry \& Li (1990) have compared spectra obtained from both calibrated and uncalibrated wires and found the differences to be negligible, i.e. the spectral shapes for voltage fluctuations are close to velocity fluctuations. The signals were sampled at three different sampling rates to improve the frequency bandwidth of the spectrum at low frequencies and were low-pass filtered at less than half the digital sampling rate to avoid aliasing of the measured spectrum. The three resulting spectral files were matched and joined to form a single spectral file which was then smoothed. To transform the spectral argument from frequency, $f$, to streamwise wavenumber, $k_{1}$, Taylor's hypothesis of frozen turbulence was used, i.e. $k_{1}=2 \pi f / U_{c}$, where $U_{c}$ is the local convection velocity, which was assumed to be equal to the local mean velocity 
at a given point in the flow. In reality, there is a spread in convection velocities at a given wavenumber which must be kept in mind when analysing the spectra.

\subsection{Cone angles}

In previous studies, Perry Lim \& Henbest (1987) and Perry \& Li (1990) have discussed the concept of a cone angle. The cone angle, $\theta_{c}$, will be defined here as the included angle in the plane of the $\mathrm{X}$-wire of an imaginary cone (assumed to be symmetric) in which virtually all inferred velocity vectors fall. If $\theta_{c}$ exceeds what will be called the critical cone angle then incorrect values of inferred turbulence intensities will result. This is where a flying hot wire can be effective since the forward bias velocity of the flying wire reduces the effective angle as seen by the $\mathrm{X}$-wire. In general the value of the critical cone angle will depend on the included angle of the X-wire (which is nominally $90^{\circ}$ for the results presented here) and the calibration formulation used in evaluating the velocity vectors. Modified calibration schemes incorporating yawresponse effects will effectively increase the allowable critical cone angle for an X-wire system. Such schemes have been used by Browne, Antonia \& Chua (1989) for jet flow, while Willmarth \& Bogar (1977) also used a similar technique for near-wall boundary layer flow.

Estimating cone angles can be simply done by considering the p.d.f. of the measured velocity vector angles. The definition adopted here is outlined as follows. Firstly, an instantaneous velocity vector angle, $\theta_{i}$, is defined in the $(x, z)$-plane

$$
\theta_{i}=\arctan \left(W_{i} / U_{i}\right)
$$

where $U_{i}$ and $W_{i}$ are instantaneous total velocities in the streamwise and normal directions. The cone angle is then assumed to be given by

$$
\theta_{c}=2\left(\left|\mu_{c}\right|+3 \sigma_{c}\right)
$$

where $\mu_{c}$ and $\sigma_{c}$ are the mean and standard deviation of the p.d.f. of measured velocity vector angles, $P\left(\theta_{i}\right)$. Typically for boundary layer flows, $\mu_{c}$ is small and the $\pm 3 \sigma_{c}$ domain includes approximately $99 \%$ of the population sample. However, it should be mentioned that tests have shown (see Marušic 1991) that vectors beyond this angle may contribute up to $6 \%$ of the Reynolds shear stresses. Hence it is important for $\theta_{c}$ to be somewhat less than the critical cone angle.

\section{Experimental results}

\subsection{Description of flows}

Two flow cases were investigated with upstream velocity, $U_{\infty}$, set nominally to 10 (10APG) and $30 \mathrm{~m} \mathrm{~s}^{-1}$ (30APG). The non-dimensional pressure gradient distributions were set up to be approximately the same for both cases and are shown in figure 2 . The boundary layer flows are seen to be initially in a zero pressure gradient condition and are then acted upon by an approximately constant adverse pressure gradient. Figure 2 also shows the location of the measuring stations where all turbulence measurements were made. Table 1 gives the relevant mean flow information for the two flow cases. All symbols have the same meaning as in Part 1 . As mentioned in $\S 1$ the effect of $\sigma$ is significant and if we compare flow case 1 (10APG) with interpolated data of East, Sawyer \& Nash (1979) for the same $\Pi$ values, the Reynolds shear stresses given in equilibrium and non-equilibrium flows are quite different as seen in figure 3 . The fluctuating velocity components will be designated $u_{1}, u_{2}$ and $u_{3}$ for the streamwise, spanwise and wall-normal directions respectively. 


\begin{tabular}{|c|c|c|c|c|c|c|c|c|c|}
\hline$x(\mathrm{~mm})$ & Symbol & $\Pi$ & $S$ & $\zeta$ & $\beta$ & $\sigma$ & $H$ & $R_{\theta}$ & $K_{\tau}$ \\
\hline 1200 & 0 & 0.42 & 23.6 & 0.15 & $\approx 0.0$ & -0.14 & 1.43 & 2206 & 1028 \\
\hline 1800 & $\nabla$ & 0.68 & 25.4 & 0.94 & 0.65 & -0.62 & 1.44 & 3153 & 1203 \\
\hline 2240 & $\square$ & 1.19 & 28.1 & 2.18 & 1.45 & -0.99 & 1.49 & 4155 & 1234 \\
\hline 2640 & $\triangle$ & 1.87 & 31.5 & 4.64 & 2.90 & -1.18 & 1.58 & 5395 & 1265 \\
\hline 2880 & $\triangleleft$ & 2.46 & 34.5 & 8.01 & 4.48 & -1.32 & 1.64 & 6395 & 1280 \\
\hline 3080 & 0 & 3.23 & 38.4 & 15.32 & 7.16 & -1.56 & 1.73 & 7257 & 1253 \\
\hline (30APG) & & & & & & & & & \\
\hline 1200 & 0 & 0.49 & 26.4 & 0.32 & $\approx 0.0$ & -0.36 & 1.40 & 6430 & 2757 \\
\hline 1800 & $\nabla$ & 0.79 & 28.2 & 0.90 & 0.71 & -0.58 & 1.41 & 8588 & 3003 \\
\hline 2240 & $\square$ & 1.12 & 30.1 & 2.09 & 1.39 & -1.19 & 1.44 & 10997 & 3243 \\
\hline 2640 & $\Delta$ & 1.65 & 32.9 & 3.98 & 2.74 & -1.30 & 1.49 & 14209 & 3427 \\
\hline 2880 & $\triangleleft$ & 2.10 & 35.2 & 6.16 & 3.96 & -1.42 & 1.54 & 16584 & 3503 \\
\hline 3080 & $\diamond$ & 2.68 & 38.1 & 10.52 & 6.07 & -1.58 & 1.60 & 19133 & 3515 \\
\hline
\end{tabular}

TABLE 1. Mean flow parameters for authors' data flow cases. Here $\Pi$ is the Coles wake factor, $S=U_{1} / U_{\tau}, \zeta=S \delta_{c} \mathrm{~d} \Pi / \mathrm{d} x$ where $\delta_{c}$ is the boundary layer thickness, $\beta=\left(\delta^{*} / \tau_{0}\right)(\mathrm{d} P / \mathrm{d} x), \sigma$ is the ratio of the shear stress contribution from $\mathrm{d} \Pi / \mathrm{d} x$ effects to total shear stress at $z / \delta_{c}=0.5$, e.g. if $\sigma=0$ we have equilibrium or quasi-equilibrium and if $|\sigma|=O(1)$ we are far from equilibrium; $H=\delta^{*} / \theta, R_{\theta}=\theta U_{1} / v$ and $K_{\tau}=\delta_{c} U_{\tau} / v$. Here $\theta$ is the momentum thickness, $\delta^{*}$ is the displacement thickness and $\tau_{0}$ is the wall shear stress.

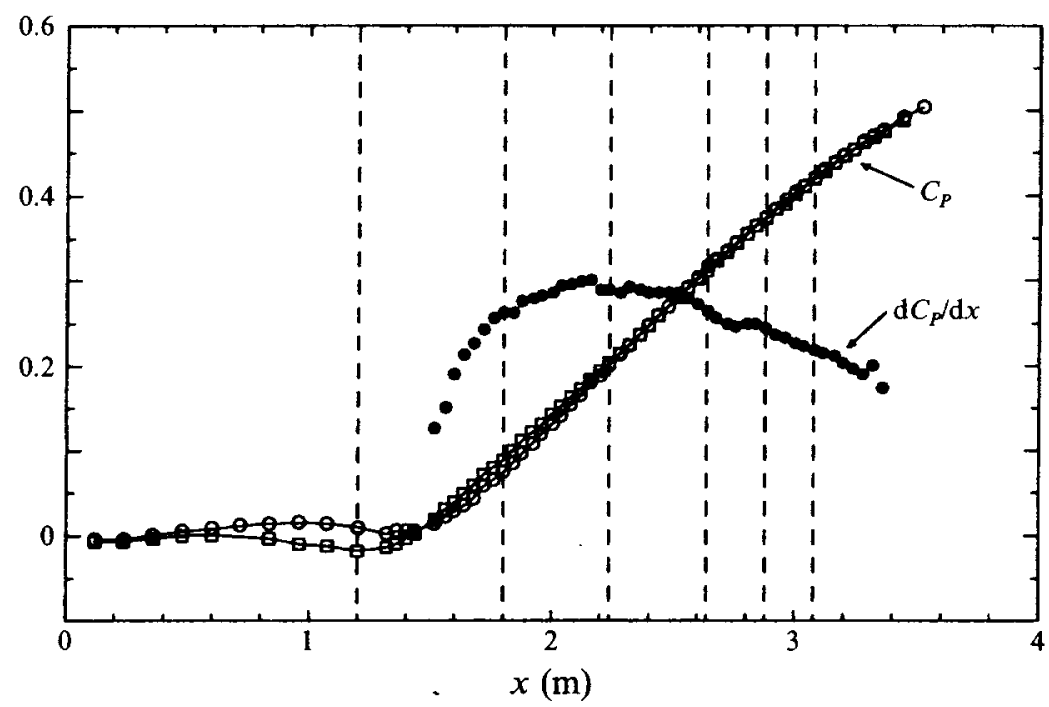

FIGURE 2. Streamwise non dimensional pressure distributions. Circles correspond to flow case 1 (10APG); squares correspond to flow case 2 (30APG). Dashed lines indicate the position of measuring stations.

\subsection{Comparison of Reynolds stresses with model}

As stated in Part 1, the theoretical predictions are valid only beyond the viscous buffer zone and that all experimental measurements were made beyond this buffer zone. The condition $z / \delta_{c} \rightarrow 0$ is meant to imply the smallest value of $z / \delta_{c}$ we can have without intruding into the buffer zone. Figure 4 shows a comparison between measured shear stresses for flow case 1 and calculated stresses using the tabulated values of $S, \Pi, \beta$ and $\zeta$ and momentum equation (15) in Part 1. Data obtained from 


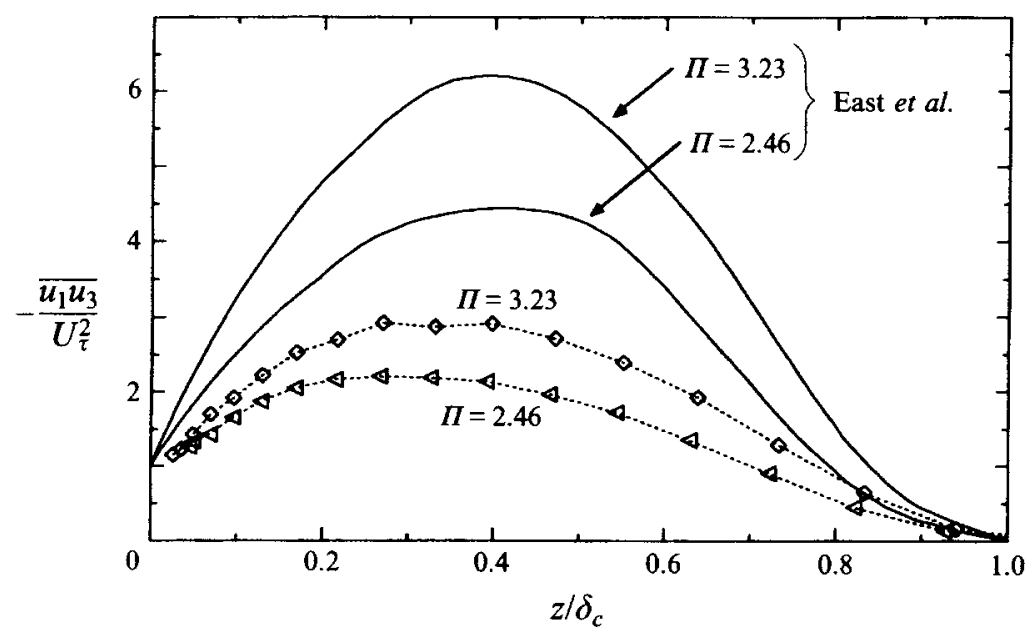

FIGURE 3. Comparison of authors' typical non-equilibrium data for $\Pi=2.46,3.23$ (10APG) with interpolated data for the same values of $\Pi$ for the equilibrium flow of East et al.

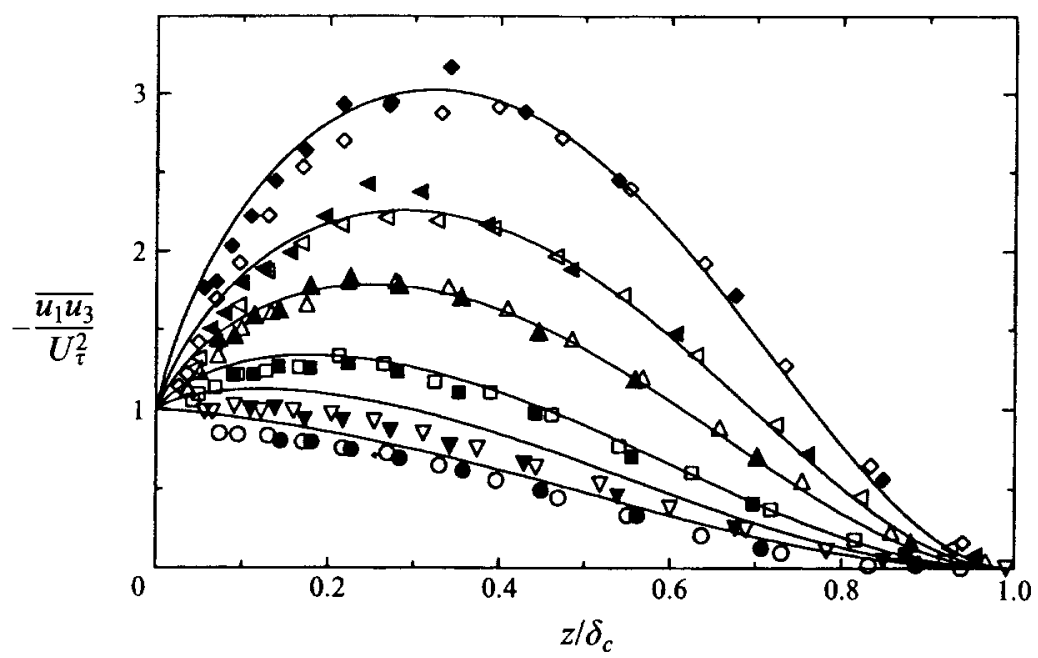

Figure 4. Reynolds shear stress profiles as described by equation (15) Part 1 using parameters given in table 1 for flow case 1 (10APG). Shaded symbols are for flying hot-wire measurements; unshaded symbols are for the corresponding stationary wire measurements.

both flying (shaded symbols) and stationary wires (unshaded symbols) are shown. The agreement is seen to be quite satisfactory. This confirms to some extent that the law of the wall and the law of the wake are excellent fits to the data and that the flow is approximately two-dimensional in the mean. It also supports the assumption that streamwise gradients of normal stresses are negligible.

Figure 5 shows the normal components of Reynolds stress, including stationary and flying measurements, compared to theoretical curves for the case of using ' $\Pi$-shaped' (figure 6, Part 1) type-A eddies alone. The computational scheme is as described in figure 17 Part 1. As was the case for the equilibrium data of East et al. (1979), this model is clearly inadequate.

Figure 6 shows the greatly improved results obtained using both type-A and type-B eddies using the formulation given in figure 18 Part 1 with eddy shapes shown in 

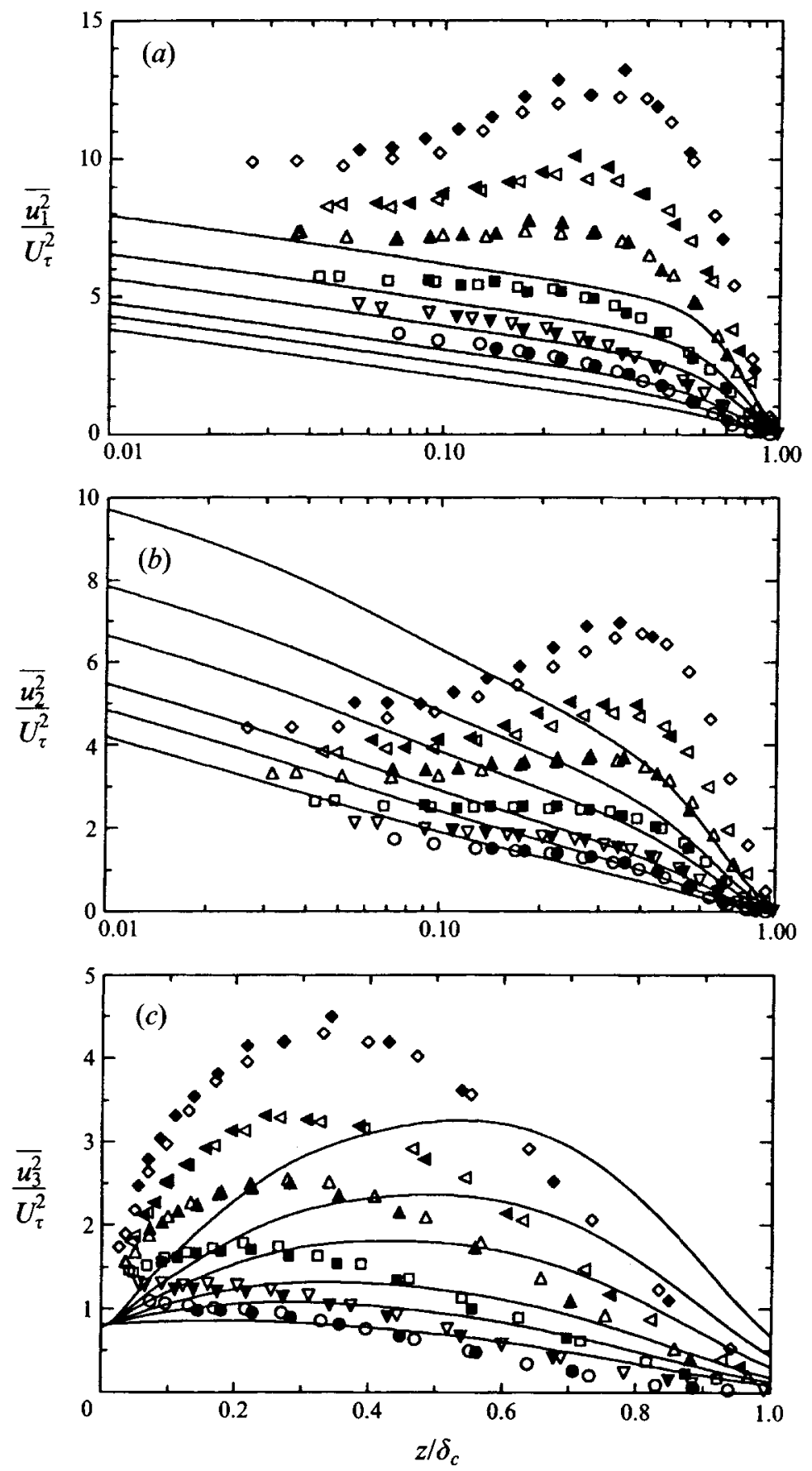

FIGURE 5. Reynolds normal stresses for flow case 1 (10APG) compared to formulation (36) of Part 1 using a type-A ' $\Pi$-shaped' eddy structure alone.

figure 13 Part 1 . The separate contributions from type-A (wall eddies) and type-B (wake eddies) structures and the composite profiles are shown for a typical case $(\Pi=2.46)$ in figure 7 .

Figures 8 and 9 show the corresponding calculations and data for the authors' 

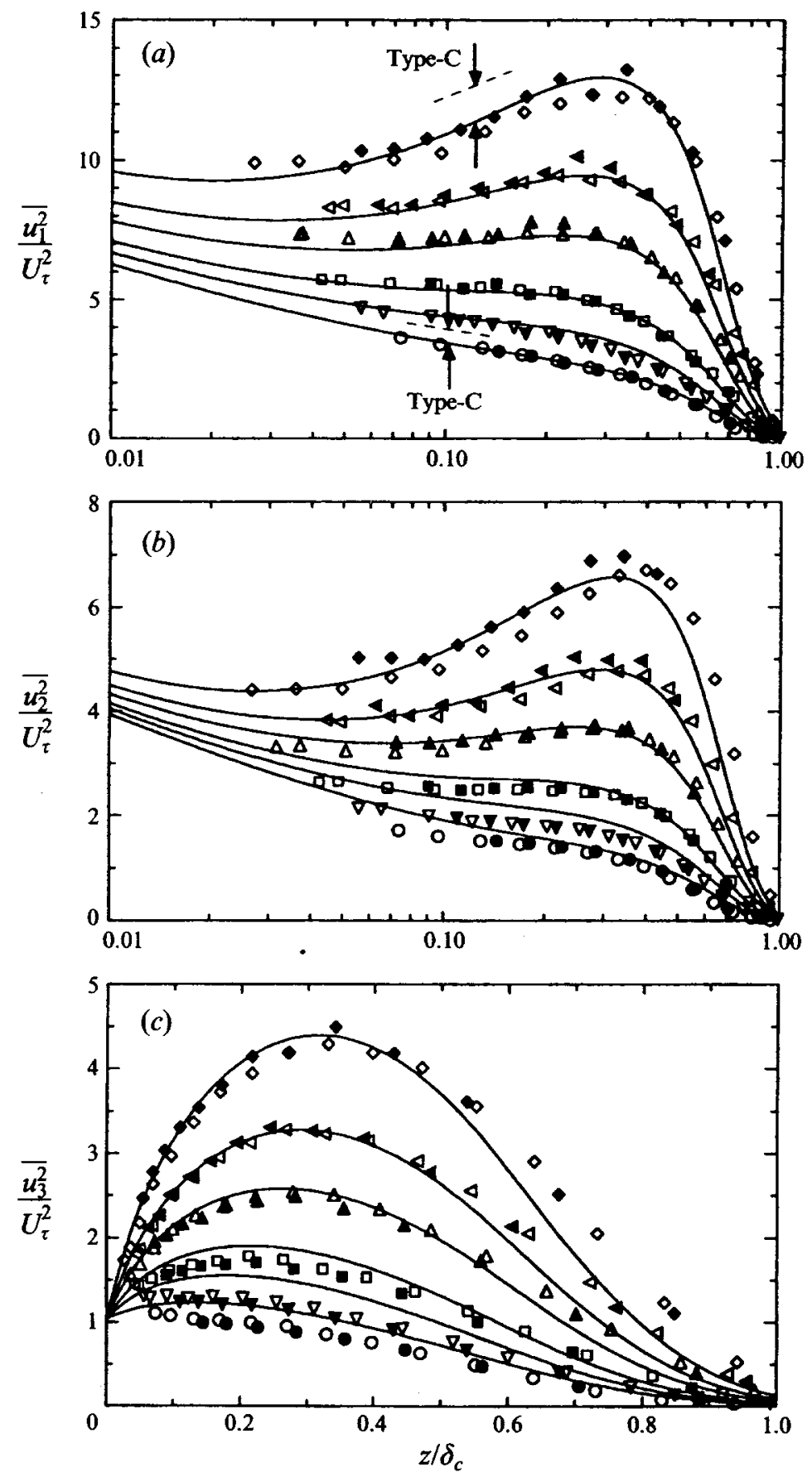

FIGURE 6. Reynolds normal stresses for flow case 1 (10APG) compared to formulation (50) Part 1 using type-A and type-B eddies as shown in figure 13 Part 1. Short broken lines indicate the estimated contribution missing from the model due to type-C detached eddy structures (including Kolmogorov contributions). 

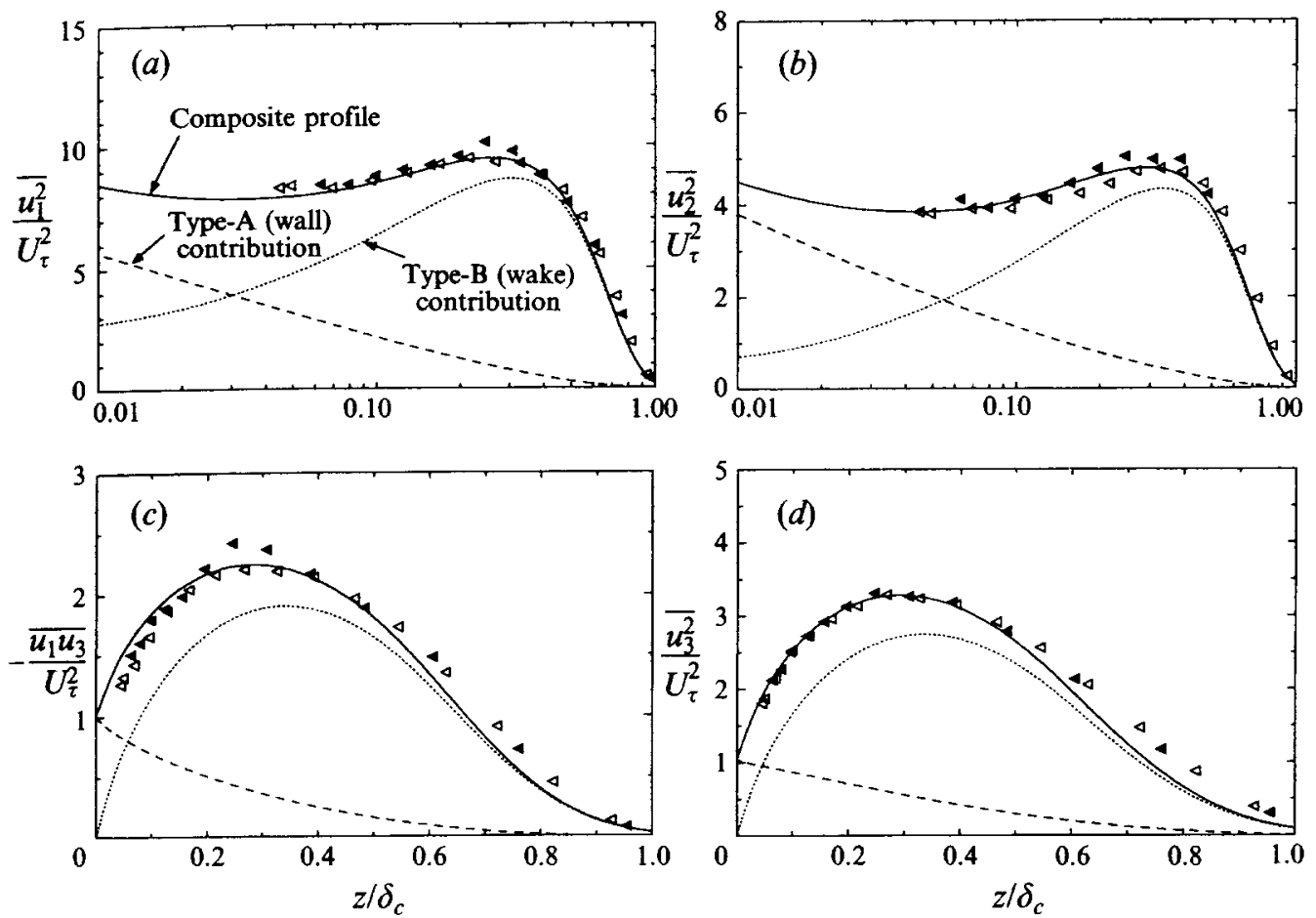

FIGURE 7. Reynolds stresses for flow case $\Pi=2.46(10 \mathrm{APG}, x=2080 \mathrm{~mm})$. Lines are from formulation (50) Part 1 using type-A and type-B eddies shown in figure 13 Part 1 with composite and individual type- $A$ and type- $B$ contributions.

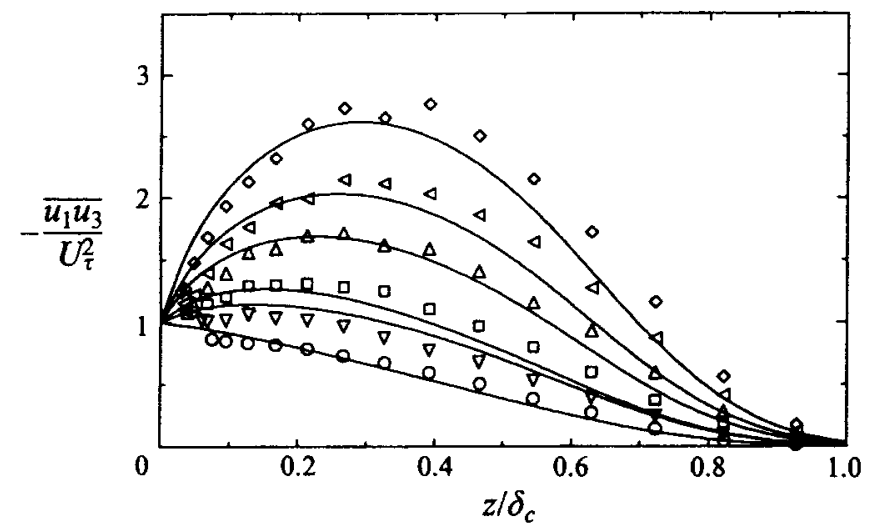

FIGURE 8. Reynolds shear stress profiles as described by equation (15) Part 1 using parameters given in table 1 for flow case 2 (30APG).

flow case 2 (30APG). (Here only stationary wire measurements were taken). The agreement with the computations again appears to be reasonable.

\subsection{Cone angles}

The comparison of flying versus stationary wire measurements shown in the previous figures indicates that in the $(x, z)$-plane no significant differences between the stationary and flying measurements are evident. This applies to all except perhaps 

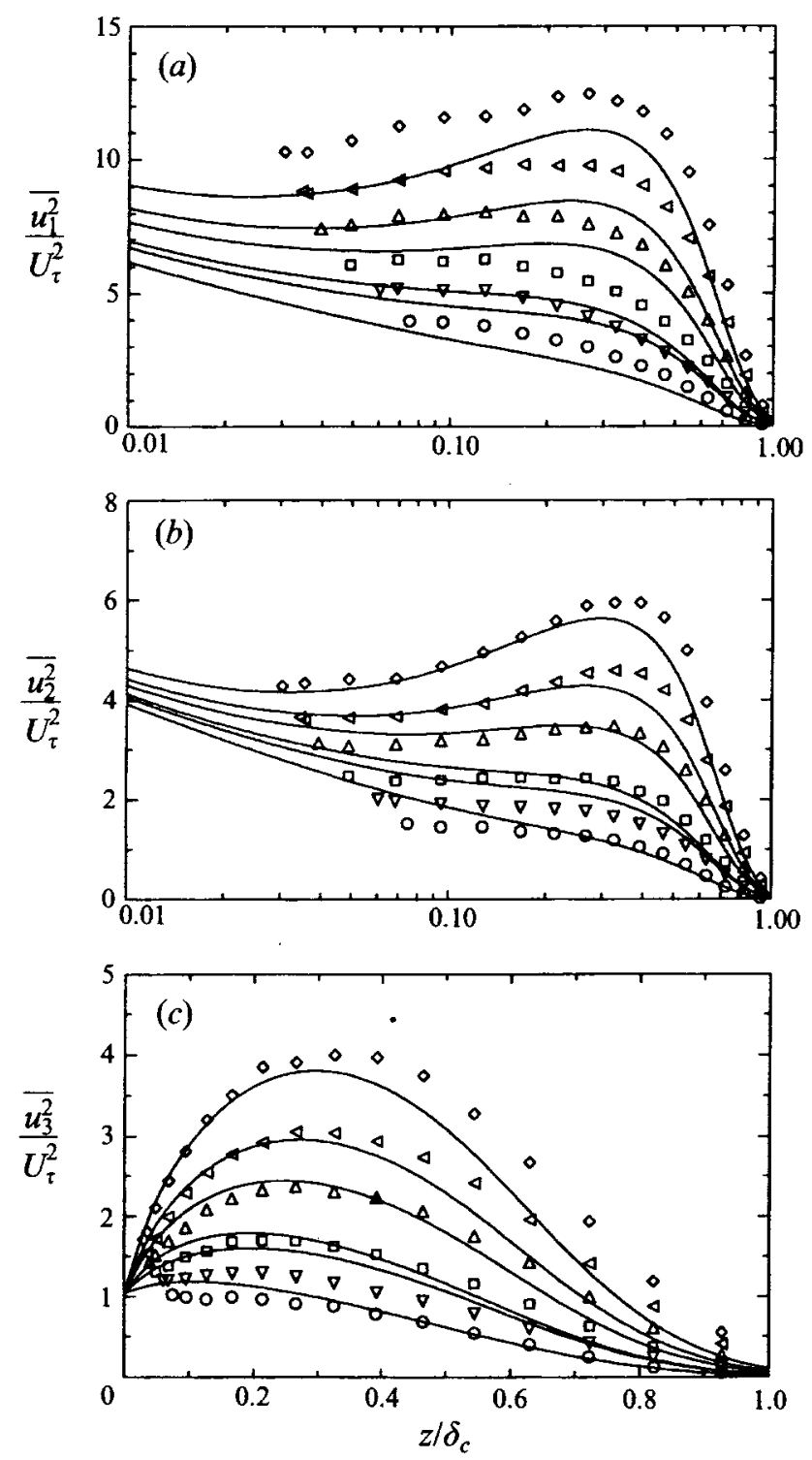

FIGURE 9. Reynolds normal stresses for flow case 2 (10APG) compared to formulation (50) Part 1 using type-A and type-B eddies as shown in figure 13 Part 1.

the $\Pi=3.23$ flow where a small departure is suggested. In the $(x, y)$-plane, the $\overline{u_{2}^{2}}$ data show clear differences for the highest $\Pi$-value stations. The difference is beyond any estimated errors in measuring $\overline{u_{2}^{2}}$ as determined from the dynamic calibration procedure. Therefore the stationary measurements are concluded to be suffering from cone-angle problems and thus underestimate the true turbulent intensities. Figure $10(a)$ shows the measured cone angles for this flow case. As suspected, the cone angles for the $(x, y)$-plane are seen to be typically more than $10^{\circ}$ larger as compared to the $(x, z)$-plane.

From the results it would seem that the critical cone angle for the $\mathrm{X}$-wire and its calibration procedure is approximately $40-45^{\circ}$ whereas the included angle of the 
(a)

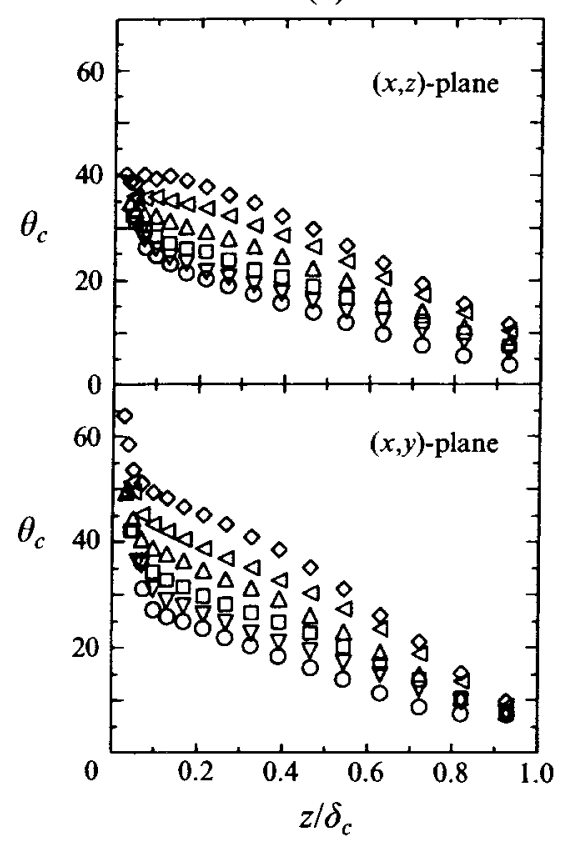

(b)

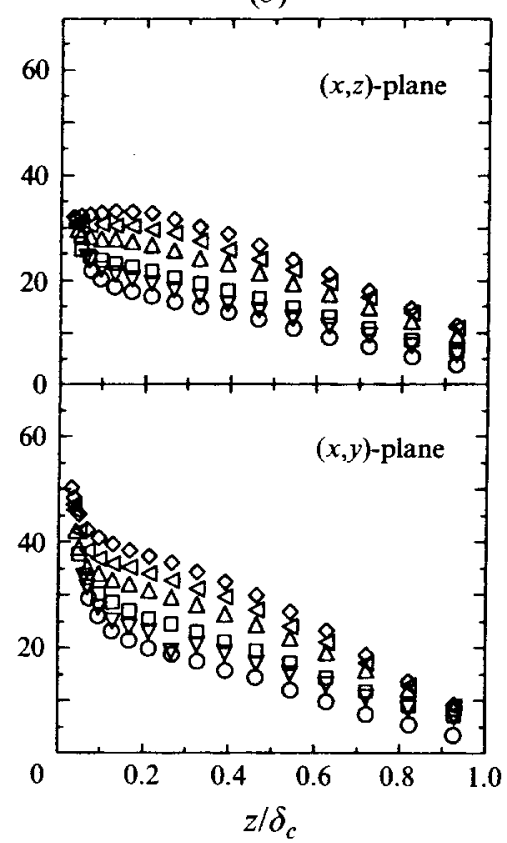

FIGURE 10. Cone angles for $(a)$ flow case 1 (10APG), (b) flow case 2 (30APG). Symbols as in table 1 .

wires is approximately $90^{\circ}$. All measurements with cone angles above this value seem to give erroneous readings. Marušic \& Perry (1992) give further details concerning cone-angle problems.

In order to determine if the flying stress measurements are in fact correct, a check of the flying hot-wire cone angles is required to ensure that they are below the critical cone angle. (If this is not the case then the flying velocity of the sled would need to be increased). Figure 11 shows the flying cone angles taken in the $(x, y)$-plane at $x=3080 \mathrm{~mm}$. Reassuringly all angles are seen to be well below the critical cone-angle threshold.

Figure $10(b)$ shows the corresponding cone angle measurements for the $30 \mathrm{~m} \mathrm{~s}^{-1}$ flow case. The results are seen to be within the above criterion and therefore the stationary Reynolds stress measurements should be of high quality.

\section{Comparison of computed spectra with data}

Consider again the two eddy structure model and calculation scheme given in figure 18 Part 1 . This will be applied to the power spectral density as defined by equations (42) and (43) of Part 1 and we can calculate $\Phi_{i j}\left[k_{1} z\right]$. This is the energy per unit non-dimensional streamwise wavenumber $k_{1} z$ and is normalized here such that $\int_{0}^{\infty} \Phi_{i j}\left[k_{1} z\right] \mathrm{d}\left[k_{1} z\right]=\overline{u_{i} u_{j}}$.

Figure 12 shows calculated premultiplied spectra for $\Phi_{11}\left[k_{1} z\right]$ broken up into the 'wall structure' component, 'wake structure' component and composite spectra for various $z / \delta_{c}$ for the first and last stations of the authors' flow case 1 . The complex changes in spectral shape are obvious. Figure 13 shows a comparison with data and although the data are incomplete it shows some of the trends and that the spectra 


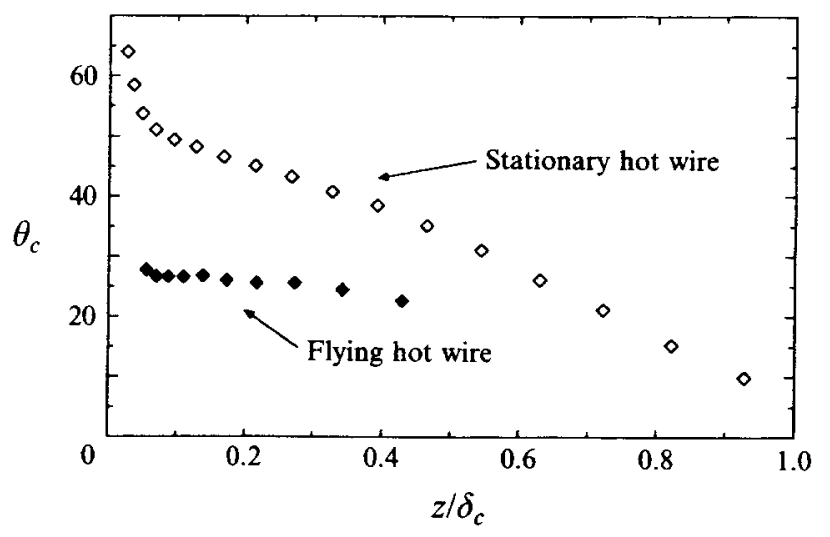

FIGURE 11. Comparison of flying (shaded) and stationary cone angles for 10APG flow case $x=3080 \mathrm{~mm} ;(x, y)$-plane. Flying velocity was about $3 \mathrm{~m} \mathrm{~s}^{-1}$.

are qualitatively and approximately quantitatively correct. Additional individual premultiplied spectra are compared with data in figures $14(a)$ and $14(b)$ for $\phi_{33}$ and it appears that we do not yet know the correct eddy shape. Further comparisons of spectra are given in Perry \& Marušic (1993) †. In general the model appears to give too much weighting to the high wavenumbers. Simple changes in eddy shape geometry could easily account for this difference, as illustrated with the example shown in figure $14(c)$.

As mentioned in Part 1, further structures must be included which contribute to the high-wavenumber motions. These will be referred to as type-C eddies. According to Perry, Henbest \& Chong (1986) these are detached eddies, i.e. their length scale is not related directly to their distance from the wall. The Kolmogorov inertial subrange and dissipation range consist of these detached eddies. We have no clear idea of the geometry of these eddies except that (according to conventional wisdom) they are locally isotropic at the high-wavenumber end of the spectrum from a statisical viewpoint. For the model outlined here to be complete, these structures must be added in using known similarity laws. Fortunately, for the fully turbulent wall region (where the logarithmic law of the wall is valid) there are guiding similarity laws together with the reasonably valid assumption that energy dissipation is in balance with energy production, see Townsend (1961). This enables the Kolmogorov length and velocity scales to be related to 'wall variables' such as $U_{\tau}$, the friction velocity and the distance $z$ normal to their wall, see Perry et al. (1986). Outside the fully turbulent wall region there is no reliable way of computing the dissipation and so Kolmogorov scaling cannot be related simply to the boundary layer variables. This remains one of the problems yet to be solved for completing the model.

Returning to the attached eddies, the authors have experimented with varying the aspect ratio and other geometric parameters of type-A eddies and it is not difficult to change the spectral shape to agree more closely with experiment. Figure $14(c)$ shows an example with $\Phi_{33}\left[k_{1} z\right]$ which for $z / \delta_{c}$ sufficiently small depends only on the wall structure eddies which are assumed here to be $\Lambda$ eddies.

It is difficult to decide on whether or not experimental data here and in past work had a sufficiently high Reynolds number to allow $z / \delta_{c}$ to become sufficiently small

$\dagger$ In this report by Perry \& Marušic (1993) the computed spectra contain a software error: the wavenumbers need to be multiplied by a factor of $\pi$. For a logarithmic abscissa this would result in a bodily shift towards the high wavenumber without any change of shape. 
(a)

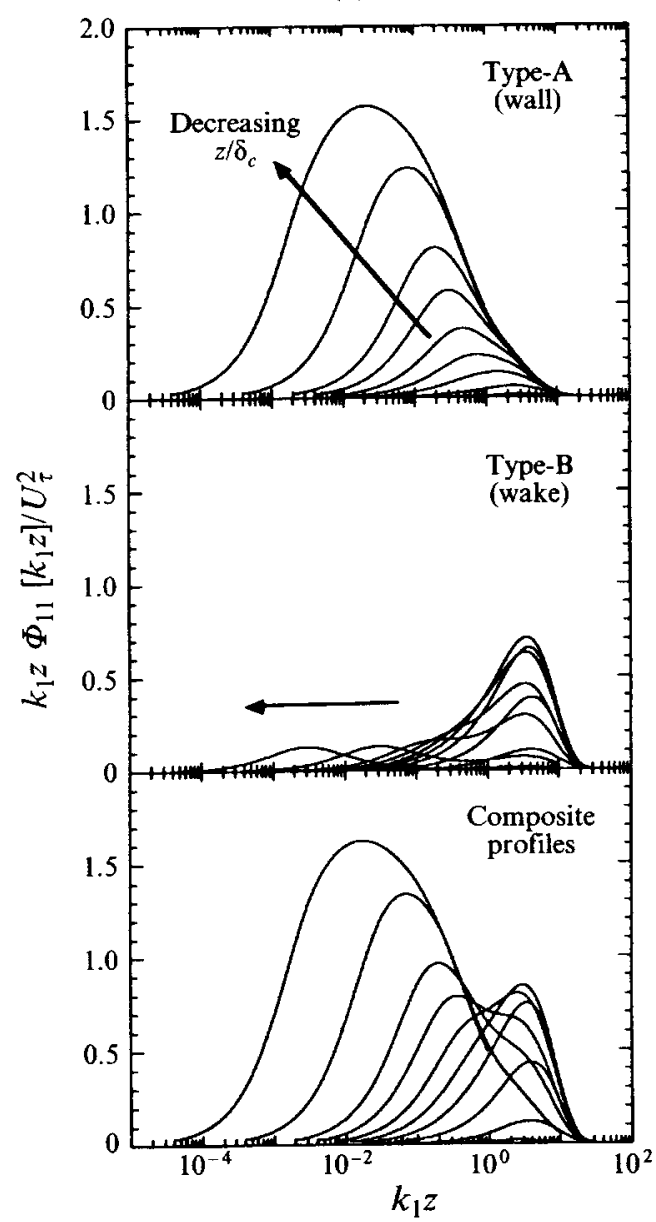

(b)

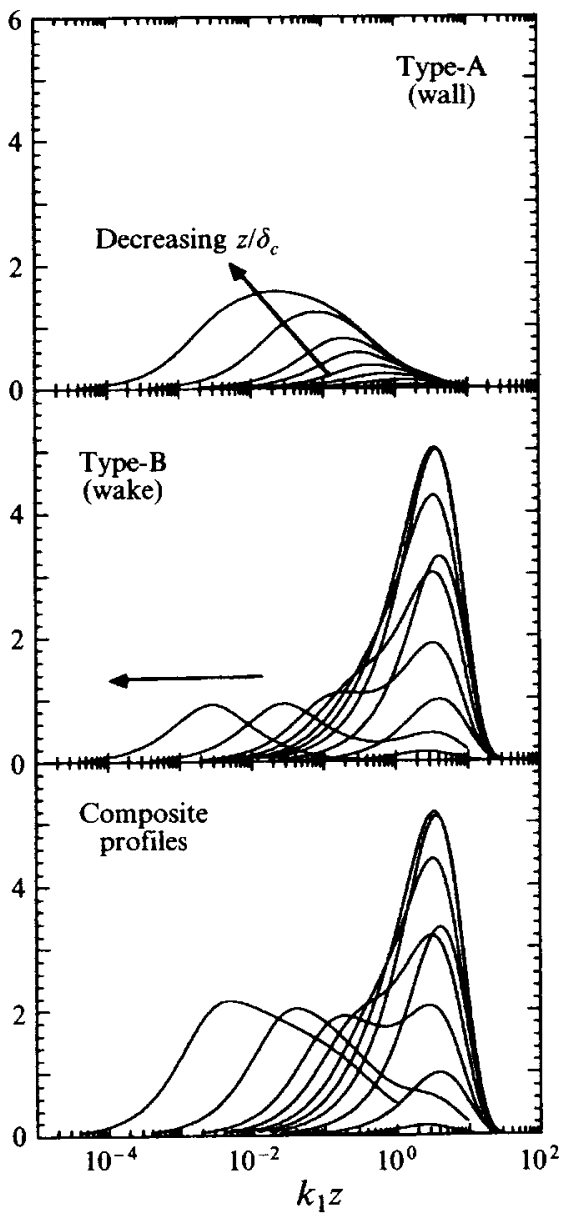

FiguRE 12. Premultiplied streamwise spectra from model showing individual type-A and type-B contributions. (a) Computed for $\Pi=0.42$ flow (10APG, approximately ZPG flow). (b) $\Pi=3.23$ (10APG) flow case. $z / \delta_{c}=0.001,0.01,0.05,0.10,0.17,0.27,0.39,0.54,0.72,0.93$.

to reach the asymptotic -1 power law for the power spectral densities $\Phi_{11}$ and $\Phi_{22}$ as proposed by Perry and Abel (1977) and Perry et al. (1986). Figure 15(a) shows the premultiplied spectra for the $\Lambda$ eddies used in the earlier calculation. One needs to go to extremely low $z / \delta_{c}$ to reach the asymptotic -1 law and the associated constant $A_{1}$ in the logarithmic law for $\overline{u_{1}^{2}} / U_{\tau}^{2}$ given in equation (54) Part 1 is $A_{1}=1.65$. If we use $\Pi$-shaped eddies with the geometry shown in figure 6 Part 1 we obtain the -1 law occurring earlier as $z / \delta_{c}$ decreases and $A_{1} \approx 0.9$ as shown in figure $15(b)$. The value of $A_{1}=1.01$ was used by Perry \& $\mathrm{Li}(1990)$ and so it could be that the actual type-A eddy shape is somewhere between a $\Pi$ and $\Lambda$ eddy. This has not yet been tried in the model. Figure 15(c) shows how the 'wake component' changes for changing streamwise distance for an extremely low value of $z / \delta_{c}$.

The basic picture put forward by Perry $\& \mathrm{Li}(1990)$ for zero pressure gradient layers remains much the same for pressure gradient layers, with modified interpretations as given in this paper. Figure 16 shows the spectra in the fully turbulent wall region. It 
(a)

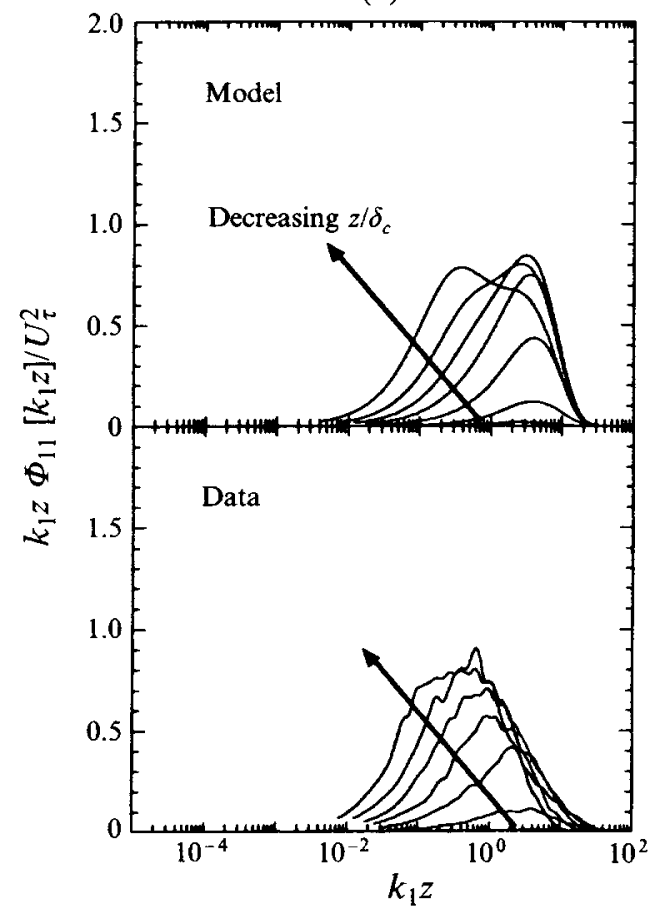

(b)

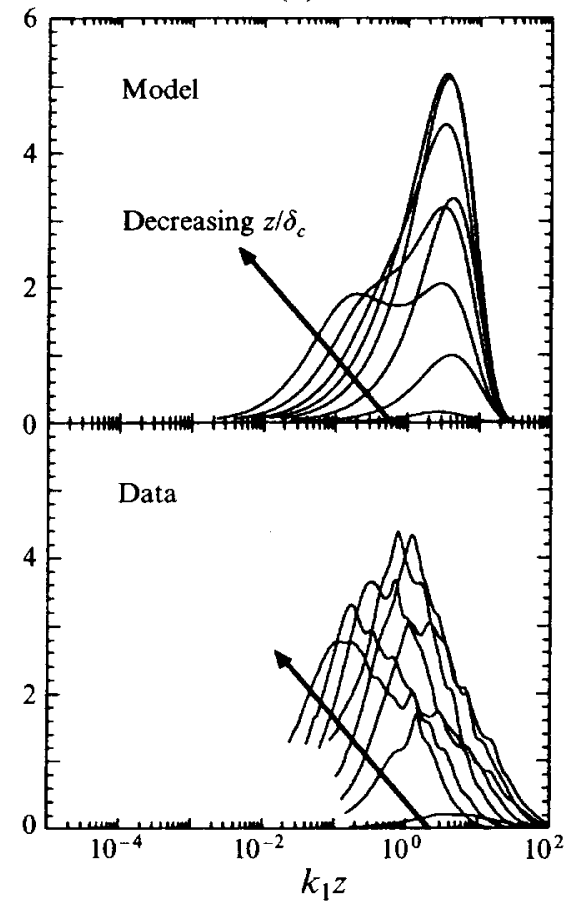

FigURE 13. Premultiplied streamwise spectra from model and authors' data. (a) $\Pi=0.42$ (10APG) flow case. $z / \delta_{c}=0.10,0.17,0.27,0.39,0.54,0.72,0.93$. (b) $\Pi=3.23$ (10APG) flow case, as in (a) with extra level $z / \delta_{c}=0.05$.

appears that the low-wavenumber bump contributed from the Coles wake component is much smaller for zero pressure gradient layers than assumed by Perry \& $\mathrm{Li} \mathrm{(1990)}$ and that the Kolmogorov region commences at $k_{1} z=N \approx 20$ rather then $N=2.5$ as suggested by Perry \& $\mathrm{Li}(1990)$. Their value for $N$ was judged from $\log -\log$ plots rather than from log-linear plots. However, all the usual scaling laws as enunciated by Perry \& Abell (1977), Perry et al. (1986) and Perry \& Li are still applicable and these are shown schematically in figure 16. In figure 16 it is seen that extra energy must be added by the type-C eddies. These eddies are the locally isotropic Kolmogorov eddies and other detached eddies.

The area contributed by type-C eddies is given by $R_{1}$ less the missing energy $V\left[z_{+}\right]$ from the Kolmogorov viscous cut-off. Hence $\overline{u_{1}^{2}} / U_{\tau}^{2}$ and the other normal stress components are given by

$$
\left.\begin{array}{l}
\overline{u_{1}^{2}} / U_{\tau}^{2}=Q_{1}[\Pi, \zeta, S]+H_{1}-A_{1} \log \left[z / \delta_{c}\right]+R_{1}-V\left[z_{+}\right], \\
\overline{u_{2}^{2}} / U_{\tau}^{2}=Q_{2}[\Pi, \zeta, S]+H_{2}-A_{2} \log \left[z / \delta_{c}\right]+R_{2}-V\left[z_{+}\right], \\
\overline{u_{3}^{2}} / U_{\tau}^{2}=K_{3}+R_{3}-V\left[z_{+}\right],
\end{array}\right\}
$$

where $H_{1}, A_{1}, R_{1}, H_{2}, A_{2}, R_{2}, K_{3}$ and $R_{3}$ are universal constants, $V\left[z_{+}\right]$is a universal function and $z_{+}=z U_{\tau} / v . Q_{1}$ and $Q_{2}$ depend on external flow conditions. For equilibrium and quasi-equilibrium layers $Q_{1}$ and $Q_{2}$ are functions only of $\Pi$ if the hypotheses put forward by Perry, et al. (1994) are correct.

$V\left[z_{+}\right]$has been computed using the Kovasznay (1948) formulation of the Kol- 

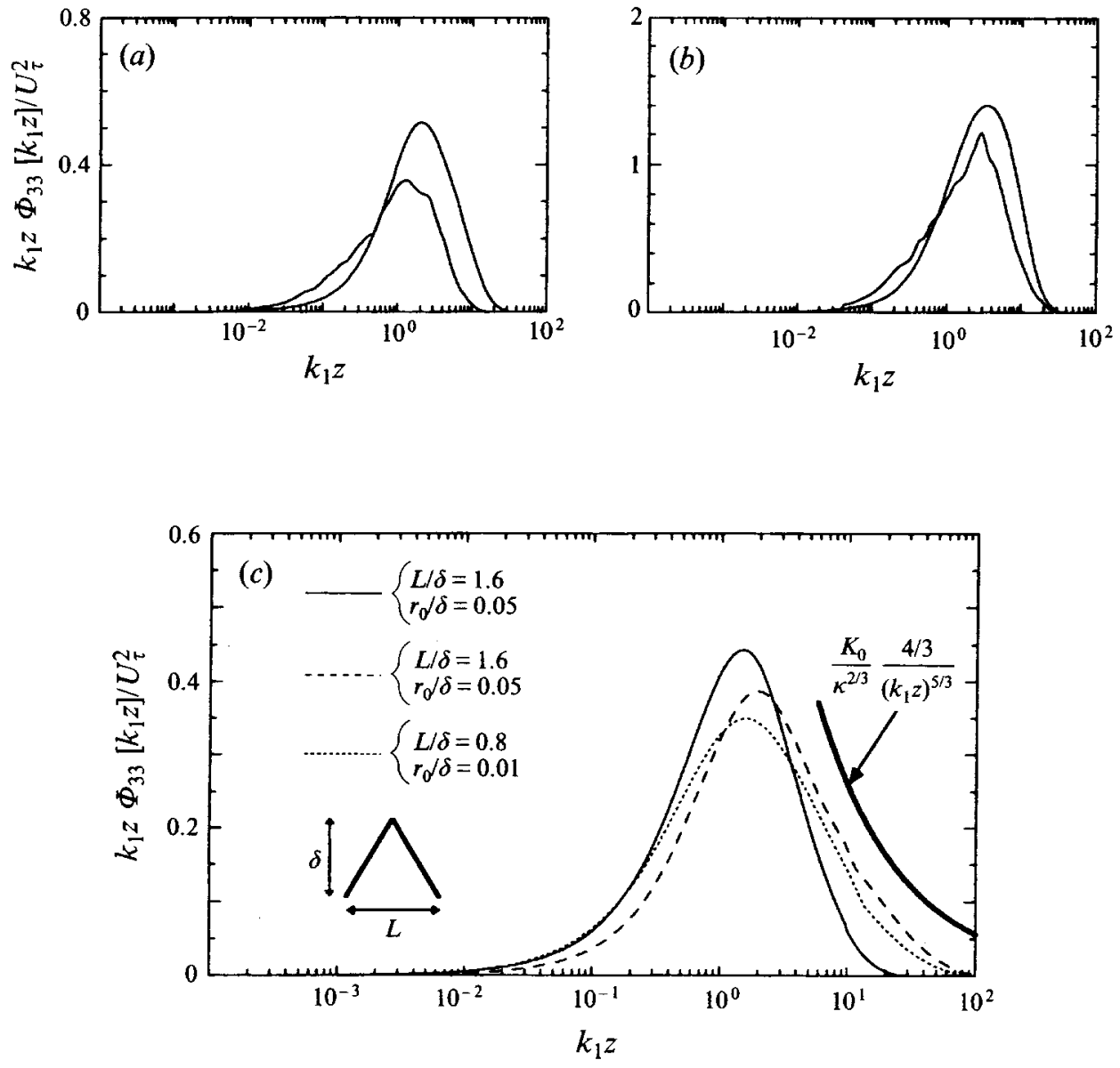

FIGURE 14. Premultiplied normal spectra $(a, b)$ : model and data (10APG) for $z / \delta_{c}=0.10$, and $(a)$ $\Pi=0.42$, (b) $\Pi=3.23$. (c) Premultiplied normal spectra for type-A $\Lambda$ eddy for varying aspect ratio and vortex rod diameter. $z / \delta_{c}=0.01$ (at this position the contribution should be purely from the wall component). Heavy line is the Kolmogorov $-5 / 3$ law $\left(K_{0}=0.5\right)$. Gaussian vorticity distribution is assumed in the vortex tubes.

mogorov inertial and dissipation subranges by Perry \& Li (1990) (their equation 21) and recently by colleague Dr S. Hafez at Melbourne. It is given by the interpolation formula

$$
V\left[z_{+}\right]=5.58\left(1-z_{+}{ }^{-0.4}\right) z_{+}{ }^{-0.5},
$$

and is valid for $O(100) \leqslant z_{+} \leqslant \infty$.

\subsection{Difficulties with type- $C$ eddies}

As can be seen from figure 16, the boundary between type- $A$ and type-C eddies is known as long as we know the precise eddy shapes for type-A. These type-C eddies, of which the Kolmogorov region is a subset, will contribute an unknown extra amount of energy to the normal stresses. Until this amount can be isolated, it is going to be difficult even to know the approximate eddy shapes. An upper bound estimate of the type- $C$ eddies contribution has been made using the chosen $\Lambda$ vortex for the type- $A$ eddies, and the error incurred by ignoring type- $\mathrm{C}$ eddy energy from the model is 


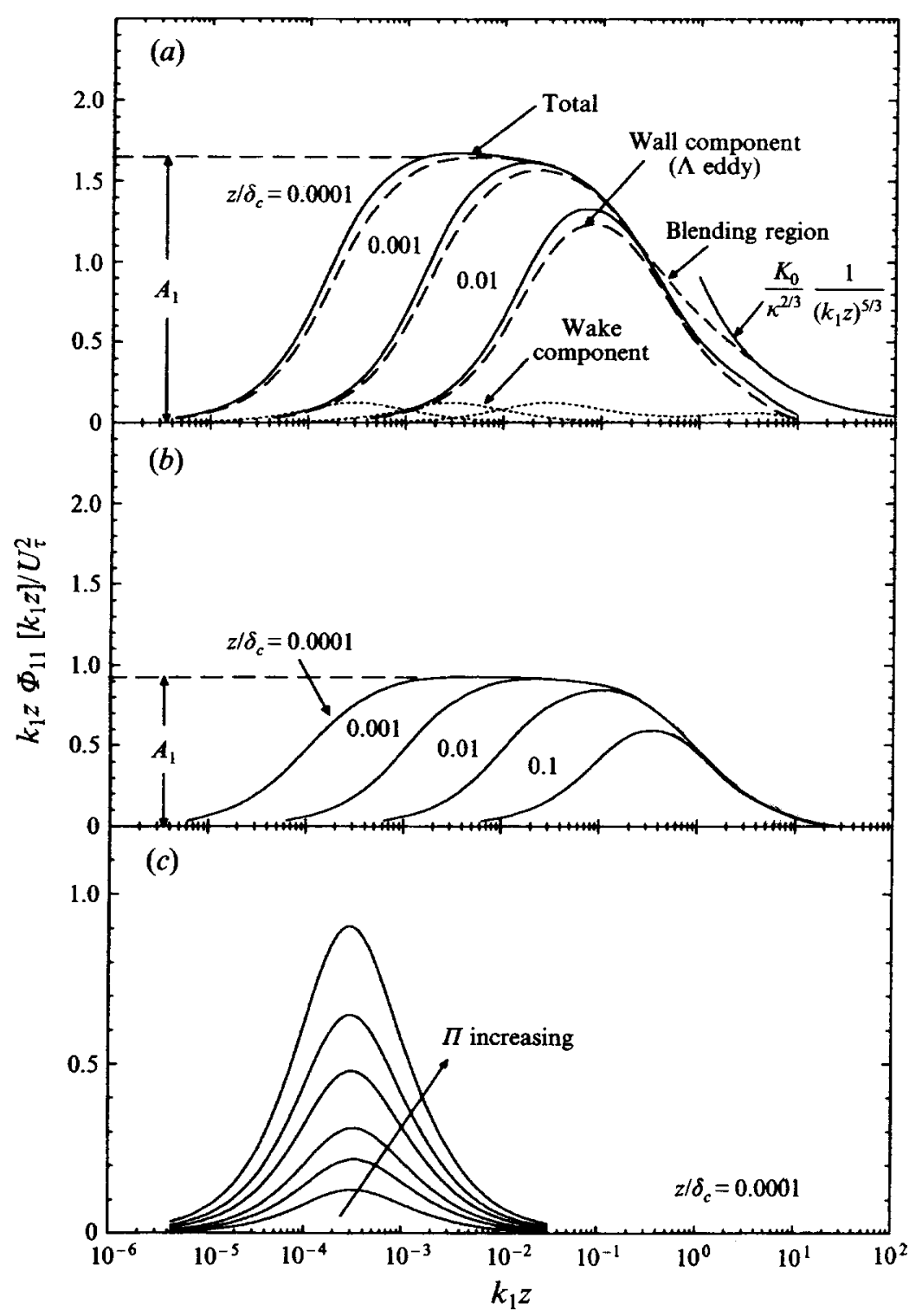

Figure 15. Premultiplied streamwise spectra showing type-A and type-B components for very small values of $z / \delta_{c}$. (a) Approximate ZPG flow $(\Pi=0.42$ ) with type-A $\Lambda$ eddies. (b) Type-A $\Pi$ eddy contribution alone. (c) Type-B component ( $\Pi$ from 0.42 to 3.23 . See table $1,10 \mathrm{APG}$ ).

indicated in figure $6(a)$. The extra energy from type-C eddies is probably of the same order for all three components of normal stresses.

\section{Conclusions and discussion}

From work carried out here, it appears that two distinct sets of attached eddies are necessary for describing most of the energy-containing motions and Reynolds shear stresses in boundary layers. The two types are distinguished by quite different conditions at the boundary. Type-A eddies are $\Lambda, \Pi$ or 'horseshoe' types and have 


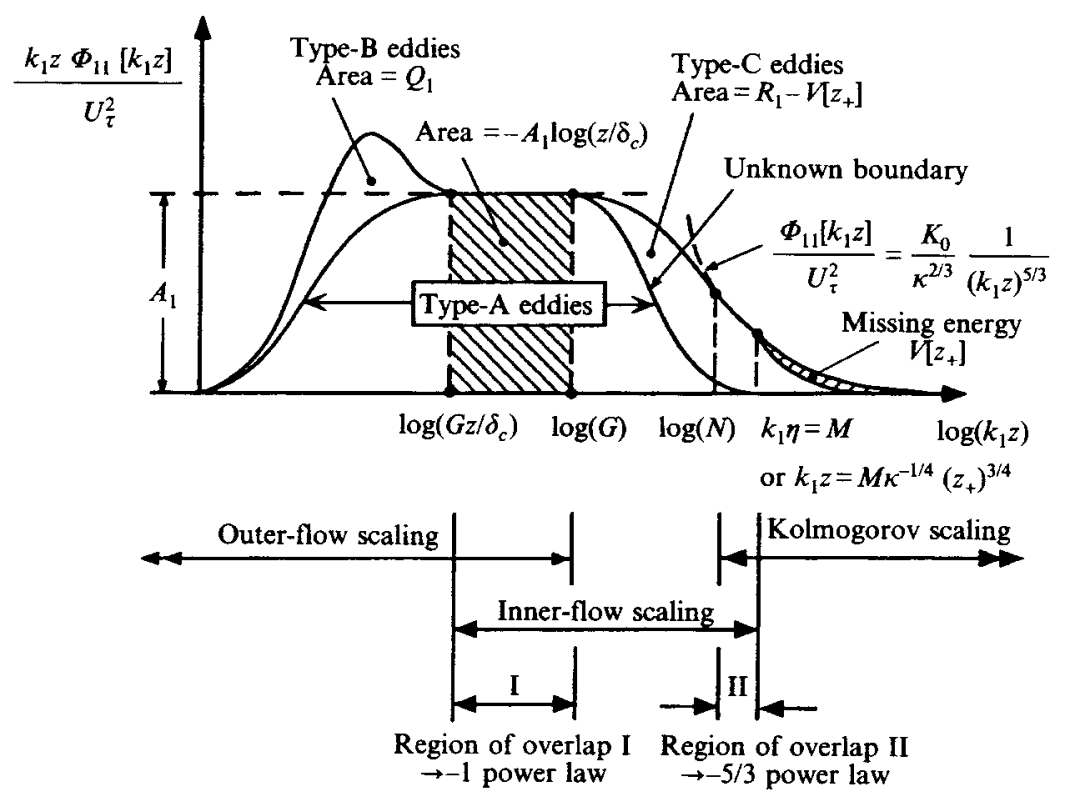

FIGURE 16. Sketch of premultiplied streamwise spectra in the turbulent wall region. $M, G$ and $N$ are universal constants. Scaling and overlap regions follow from the work of Perry \& Abell (1977) and Perry et al. (1986).

vortex lines which reach the wall, whereas with type-B eddies the vortex lines undulate in the spanwise direction and do not extend to the wall. Type-A eddies produce a finite Reynolds shear stress at the wall (at least outside the viscous zone) whereas type-B eddies give a corresponding Reynolds shear stress of zero at the wall.

It seems most natural (and calculations seem to bear this out) that type-A eddies are the 'wall' structures whereas type-B are the 'wake' structures and it is suggested here that this applies to both the mean flow and to all the Reynolds stress components. It seems plausible that the flow description needs only one universal eddy shape for type$A$ and another for type-B and that variations in the velocity scale and population density weighting factors, as functions of the eddy length scale, can account for all possible boundary layer states. The model appears to be applicable to both equilibrium and non-equilibrium boundary layers. The fine-scale dissipating motions and other detached motions must be included in the model for completeness and at the moment this is done empirically for the logarithmic wall region. For regions beyond this, we need to be able to predict the dissipation and there is no reliable theory for this. We need also to establish the true attached eddy shape so that we can isolate the contributions made by type-C eddies, i.e. we need to know the unknown boundary shown in figure 16 .

It is envisaged that the wall structure is universal and is the same as the 'pure wall' flow in equilibrium sink flow (where $\Pi=0$ and $\beta=-0.5$ ) as was suggested by Coles (1957) for the mean flow contributions. As for the wake structure, its properties are evaluated by a deconvolution and convolution using equations (30) and (36) of Part 1 once the Reynolds shear stress is evaluated from the momentum relationship (15). Given the parameters $\Pi, S, \beta$, and $\zeta$ then in principle all Reynolds stresses and associated spectra from the attached eddies can be found from the momentum relation (based on momentum and the mean velocity law of the wall and wake) once 
the eddy structure geometry has been fixed. No empirical constants are needed other than the eddy geometry constants and the law of the wall and wake constants.

It is tempting to suggest that the formulation should be valid through the entire range of boundary layer parameters ranging from $\Pi=0$ (pure wall flow) to $\Pi=\infty$ (pure wake flow). The $\Pi=\infty$ idea has not yet been pursued. Work by Dengel \& Fernholz (1990) has discouraged this type of thinking. They have experimental results which do not support the 'wall-wake' model at very high $\Pi$ without a wake function modification but this may not be a serious drawback. Also one must keep in mind the possibility of reviving the often discredited half power law of Stratford (1959) and many similar formulations which followed in the 1960s. More work is required on this aspect. In any case, the model presented here for $\Pi=0$ to say $\Pi=7$ seems very encouraging. The authors make no claim to have found the correct eddy geometries other than the gross features. Since there is an uncertainty caused by a lack of knowledge of type-C eddy contributions, there is little point in pursuing a computer-intensive systematic investigation for more precise type-A and type-B eddy shapes at this time.

The authors wish to thank the Australian Research Council for the financial support of this project. Also we acknowledge the many interesting and fruitful discussions with Professor D. Coles at GALCIT Caltech.

\section{REFERENCES}

Browne, L. W. B., Antonia, R. A. \& ChuA, L. P. 1989 Velocity vector cone angles in turbulent flows. Exps. Fluids 8, 13-16.

Clauser, F. H. 1954 Turbulent boundary layers in adverse pressure gradients. J. Aero. Sci. 21, 91-108.

CoLes, D. E. 1957 Remarks on the equilibrium turbulent boundary layer J. Aero. Sci. 24, 459-506.

Dengel, G. \& Fernholz, H. H. 1990 An experimental investigation of an incompressible turbulent boundary layer in the vicinity of separation. J. Fluid Mech. 212, 615-636.

EASt, L. F., SAwYER, W. G. \& Nash, C. R. 1979 An investigation of the structure of equilibrium turbulent boundary layers. RAE Tech. Rep. 79040.

Kovasznay, L. S. G. 1948 Spectrum of locally isotropic turbulence. J. Aero. Sci. 15, 745-753.

MARUŠÍ́, I. 1991 The structure of zero- and adverse-pressure gradient turbulent boundary layers. PhD thesis, University of Melbourne.

Marušíc, I. \& Perry, A. E. 1992 Cone angles and reynolds stresses in an adverse perssure gradient boundary layer. In Proc. 11th Australasian Fluid Mech. Conf. Hobart, Australia.

Perry, A. E. 1982 Hot-Wire Anemometry. Clarendon Press.

Perry, A. E. \& ABeLl, C. J. 1977 Asymptotic similarity of turbulence structures in smooth- and rough-walled pipes. J. Fluid Mech. 79, 785-799.

Perry, A. E., Henbest, S. M. \& Chong, M. S. 1986 A theoretical and experimental study of wall turbulence. J. Fluid Mech. 165, 163-199.

Perry, A. E. \& LI, J. D. 1990 Experimental support for the attached eddy hypothesis in zeropressure-gradient turbulent boundary layers. J. Fluid Mech. 218, 405-438.

Perry, A. E. \& Li, J. D. 1991 Theoretical and experimental studies of shear stress profiles in two dimensional turbulent boundary layers. Rep. FM-18. Dept. of Mech. Engng, University of Melbourne.

PerRy, A. E., LIM, K. L., \& HenBest, S. M. 1987 An experimental study of the turbulence structure in smooth- and rough-wall boundary layers. J. Fluid Mech. 177, 437-466.

Perry, A. E. \& Maruší́, I. 1993 A wall-wake model for the turbulence structure of boundary layers using attached eddies. Rep. FM-21. Dept. of Mech. Engng, University of Melbourne.

Perry A. E. \& Marušić, I. 1995 A wall-wake model for the turbulence structure of boundary layers. Part 1. Extension of the attached eddy hypothesis. J. Fluid Mech. 298, 361-388. 
A wall-wake model for the turbulence structure of boundary layers. Part 2407

Perry, A. E., Marušić, I. \& LI, J. D. 1994 Wall turbulence closure based on classical similarity laws and the attached eddy hypothesis. Phys. Fluids 6, 1024-1035.

StRaTFORD, B. 1959 The prediction of separation of the turbulent boundary layer. J. Fluid Mech. 5, $1-16$.

TownsEnd, A. A. 1961 Equilibrium layers and wall turbulence. J. Fluid Mech. 11, 97-120.

Townsend, A. A. 1976 The Structure of Turbulent Shear Flow. Cambridge University Press.

Watmuff, J. H., Perry, A. E. \& Chong, M. S. 1983 A flying hot-wire system. Exps. Fluids 1, 63-71.

WILlMaRTH, W. W. \& BogaR, T. J. 1977 Survey and new measurements of turbulent structure near the wall. Phys. Fluids 20, S9-S21. 


\section{University Library}

\section{- M M N E R VA A gateway to Melbourne's research publications}

Minerva Access is the Institutional Repository of The University of Melbourne

Author/s:

Marusic, I.;Perry, A. E.

Title:

A wall-wake model for the turbulence structure of boundary layers. Part 2. Further experimental support

Date:

1995

Citation:

Marusic, I. \& Perry, A. E. (1995). A wall-wake model for the turbulence structure of boundary layers. Part 2. Further experimental support. Journal of fluid mechanics, 298, 389-407.

Publication Status:

Published

Persistent Link:

http://hdl.handle.net/11343/34812 